\title{
Cerebral functional connectivity periodically (de)synchronizes with anatomical constraints
}

\author{
Raphaël Liégeois $^{1} \cdot$ Erik Ziegler $^{2}$ - Christophe Phillips ${ }^{1,2} \cdot$ Pierre Geurts $^{1,8}$. \\ Francisco Gómez ${ }^{2,3}$ - Mohamed Ali Bahri ${ }^{2}$ - B. T. Thomas Yeo ${ }^{6}$ Andrea Soddu ${ }^{4}$. \\ Audrey Vanhaudenhuyse ${ }^{5} \cdot$ Steven Laureys $^{2} \cdot$ Rodolphe Sepulchre $^{1,7}$
}

Received: 24 December 2014/ Accepted: 6 July 2015

(C) Springer-Verlag Berlin Heidelberg 2015

\begin{abstract}
This paper studies the link between restingstate functional connectivity (FC), measured by the correlations of fMRI BOLD time courses, and structural connectivity (SC), estimated through fiber tractography. Instead of a static analysis based on the correlation between SC and FC averaged over the entire fMRI time series, we propose a dynamic analysis, based on the time evolution of the correlation between SC and a suitably windowed FC. Assessing the statistical significance of the time series
\end{abstract}

Steven Laureys and Rodolphe Sepulchre contributed equally.

Electronic supplementary material The online version of this article (doi:10.1007/s00429-015-1083-y) contains supplementary material, which is available to authorized users.

Raphaël Liégeois

R.Liegeois@ulg.ac.be

Erik Ziegler

Erik.Ziegler@ulg.ac.be

Christophe Phillips

C.Phillips@ulg.ac.be

Pierre Geurts

P.Geurts@ulg.ac.be

Francisco Gómez

fagomezj@gmail.com

Mohamed Ali Bahri

M.Bahri@ulg.ac.be

B. T. Thomas Yeo

thomas.yeo@nus.edu.sg

Andrea Soddu

asoddu@uwo.ca

Audrey Vanhaudenhuyse

avanhaudenhuyse@chu.ulg.ac.be

Steven Laureys

Steven.Laureys@ulg.ac.be against random phase permutations, our data show a pronounced peak of significance for time window widths around 20-30 TR (40-60 s). Using the appropriate window width, we show that FC patterns oscillate between phases of high modularity, primarily shaped by anatomy, and phases of low modularity, primarily shaped by inter-network connectivity. Building upon recent results in dynamic FC, this emphasizes the potential role of SC as a transitory architecture between different highly connected restingstate FC patterns. Finally, we show that the regions contributing the most to these whole-brain level fluctuations of FC on the supporting anatomical architecture belong to the default mode and the executive control networks

Rodolphe Sepulchre

R.Sepulchre@eng.cam.ac.uk

1 Department of Electrical Engineering and Computer Science, University of Liège, Liège, Belgium

2 Cyclotron Research Centre, University of Liège, Liège, Belgium

3 Computer Science Department, Universidad Central de Colombia, Bogotá, Colombia

4 Department of Physics and Astronomy, Mind and Brain Institute, Western University, London, ON, Canada

5 Department of Algology and Palliative Care, University Hospital of Liége, Liége, Belgium

6 Department of Electrical and Computer Engineering, Clinical Imaging Research Centre, Singapore Institute for Neurotechnology \& Memory Networks Programme, National University of Singapore, Singapore, Singapore

7 Department of Engineering, University of Cambridge, Trumpington Street, Cambridge CB2 1PZ, UK

8 GIGA-R, University of Liège, Liège, Belgium 
suggesting that they could be capturing consciousness-related processes such as mind wandering.

Keywords Functional connectivity - Structural connectivity · Dynamics - Spontaneous activity · FMRI . DWI $\cdot$ Windowing $\cdot$ Multimodal imaging $\cdot$ Mind wandering

\section{Introduction}

The human brain shows organized spatiotemporal activity even in task-free or "resting-state" conditions which is characterized by very slow $(<0.1 \mathrm{~Hz})$ fluctuations of the fMRI Blood Oxygen Level Dependent (BOLD) signal (Gusnard et al. 2001; Greicius et al. 2003). Separate and possibly remote cerebral regions have been shown to exhibit coherent activity patterns as measured by the correlation between regional fMRI BOLD time series. This measure of so-called functional connectivity (FC) [see Friston (2011) for a review] is organized in robust resting-state networks (Beckmann et al. 2005; Damoiseaux et al. 2006; Moussa et al. 2012), and has been used to explore a range of properties such as cognition (Richiardi et al. 2011; Heine et al. 2012), emotions (Eryilmaz et al. 2011), and learning (Bassett et al. 2011). From an anatomical point of view, structural connectivity (SC) and its multi-scale spatial organization have also been characterized (Sporns et al. 2004, 2005) and linked to brain diseases (Kaiser 2013; Griffa et al. 2013; Engel et al. 2013) and genetic influences (Jahanshad et al. 2013; Ziegler et al. 2013). The relationship between SC and FC, and more particularly the way cerebral anatomy shapes neuronal functions is a question that has been addressed ever since neuroimaging techniques allowed to collect both structural and functional information [e.g., McIntosh and Gonzalez-Lima (1994)]. Different approaches have been used to tackle this question, such as direct comparison of functional and structural connectivities (Kötter and Sommer 2000; Sporns et al. 2000), graph theory (Passingham et al. 2002; Bullmore and Sporns 2009), and model-based approaches to explain the link between SC and FC (Koch et al. 2002). However, it is only recently that a clear link between SC and FC (Honey et al. 2009; van den Heuvel et al. 2009) [reviewed in Damoiseaux and Greicius (2009)] has been established, allowing for testable models (Honey et al. 2010; Deco et al. 2012). Meanwhile, the classical approach of assuming FC as constant during resting-state recordings (Bullmore and Sporns 2009; Friston 2011) has also evolved recently. We will refer to this assumption as a static analysis of FC that treats FC as a static quantity, averaging FC over the entire time series. In contrast, many recent studies have emphasized the importance of treating FC as a dynamical quantity, that is, evolving in time (Hutchison et al. 2013; Park and Friston 2013). Different tools have been proposed to introduce temporal variations into the analyses of FC, such as sliding windows (Sakoğlu et al. 2010; Bassett et al. 2011; Jones et al. 2012; Shirer et al. 2012; Allen et al. 2012; Handwerker et al. 2012), dynamic conditional correlation (Lindquist et al. 2014), single-volume co-activation patterns (CAPs) (Tagliazucchi et al. 2012; Liu and Duyn 2013; Amico et al. 2014), as well as a combination of sliding windows and other methods, such as Independent Component Analysis (Kiviniemi et al. 2011) or Principal Component Analysis (Leonardi et al. 2013). For a review of these methods, see (Hutchison et al. 2013). Using a dynamical framework, various studies have further shown that dynamical FC (dFC) can be seen as the transition between several FC patterns (Gao et al. 2010; Deco et al. 2013a; Yang et al. 2014) presenting varying network properties (Lv et al. 2013; Sidlauskaite et al. 2014; Gollo and Breakspear 2014; Shen et al. 2015). The level of variation, or flexibility of dFC between specific cerebral regions has also been studied (Bassett et al. 2011; Allen et al. 2012; Gonzalez-Castillo et al. 2014), as well as the role of anatomy in these fluctuations (Gollo et al. 2015) in the macaque cortex. Finally, many groups have explored daydreaming, or mind wandering using functional imaging. The networks implied in these processes are mainly the default mode network (Kucyi and Davis 2014; Fox et al. 2013), the executive control network (Christoff et al. 2009) as well as their interplay (Vanhaudenhuyse et al. 2011; Hasenkamp et al. 2012). The dynamical properties of mind wandering have also been studied and characteristic frequencies on the order of 0.03-0.05 Hz were found (Bastian and Sackur 2013; Vanhaudenhuyse et al. 2011). In this work, we study the dynamical correlation between $\mathrm{dFC}$ and SC using a sliding window approach and explore the role of anatomy in the fluctuations of $\mathrm{dFC}$. The first part of the paper addresses the issue of selecting a proper time window, leading to confirmatory, yet original and independent results (Allen et al. 2012; Hutchison et al. 2013; Leonardi and Van De Ville 2015). Next, motivated by recent work on the dynamic functional connectivity repertoire (Yang et al. 2014; Sidlauskaite et al. 2014) and the influence of the underlying architecture on the flexibility of dFC (Gollo et al. 2015), we explore the role of anatomy in the shaping of different FC patterns, the transition between these states, and their structure. To this end, we compute the dynamic correlation between SC and $\mathrm{dFC}$ using the appropriate sliding window width and test whether the level of SC-dFC correlation influences dFC variability or the organization of FC patterns. Based on a spatiotemporal arguments, we finally discuss the possible 
interpretation of the $\mathrm{SC}-\mathrm{dFC}$ fluctuations in terms of consciousness-related processes.

\section{Material and methods}

\section{Participants}

Data were collected from 14 healthy volunteers (age range $45 \pm 7$ years, 7 women, all right-handed). Volunteers gave their written informed consent to participate in the study, which was approved by the Ethics Committee of the Faculty of Medicine of the University of Liége.

\section{Diffusion-weighted imaging}

\section{DWI acquisition}

Data were acquired on a 3T head-only scanner (Magnetom Allegra, Siemens Medical Solutions, Erlangen, Germany) operated with the standard transmit-receive quadrature head coil. A high-resolution T1-weighted image was acquired for each subject (3D magnetization-prepared rapid gradient-echo sequence, field of view $=256 \times 240$ $\times 120 \mathrm{~mm}^{3}$, voxel size $=1 \times 1 \times 1.2 \mathrm{~mm}$ ). A single unweighted $(b=0)$ volume was acquired followed by a set of diffusion-weighted ( $b=1000)$ images using 64 noncolinear directional gradients. This sequence was repeated twice for a total of 130 volumes.

\section{Processing}

The processing pipeline was developed in Nipype (Gorgolewski et al. 2011) and has been described in more detail previously (Ziegler et al. 2013). Structural MR images were first segmented using the automated labeling of Freesurfer (Desikan et al. 2006). Segmented structural images were then further parcellated using the Lausanne 2008 atlas for a total of 1015 regions of interest (ROIs) (Cammoun et al. 2012; Hagmann et al. 2008). Diffusion-weighted images were aligned using FSL to the initial unweighted volume to correct for image distortions arising from eddy currents (Smith et al. 2004). Fractional anisotropy maps were generated, and a small number of single-fiber (high FA) voxels were used to estimate the spherical harmonic coefficients of the response function from the diffusion-weighted images (Tournier et al. 2004, 2007). Using non-negativity constrained spherical deconvolution, fiber orientation distribution (FOD) functions were obtained at each voxel. For our dataset with 64 directions, we used the maximum allowable harmonic order of 8 for both the response estimation and spherical deconvolution steps. Probabilistic tractography was performed throughout the whole brain using seeds from subject-specific white matter masks and a predefined number of tracks (Tournier et al. 2012). Fiber tracking settings were as follows: number of tracks = 300,000 , FOD amplitude cutoff for terminating tracks $=0.1$, minimum track length $=10 \mathrm{~mm}$, maximum track length $=$ $200 \mathrm{~mm}$, minimum radius of curvature $=1 \mathrm{~mm}$, tracking algorithm step size $=0.2 \mathrm{~mm}$. Using tools from Dipy (Diffusion in Python, http://nipy.sourceforge.net/dipy/), the tracks were affine-transformed into the subject's structural space and connectome mapping was performed by considering every contact point between each tract and the outlined regions of interest (Ziegler et al. 2013).

\section{Functional data}

\section{BOLD acquisition}

Three hundred multi-slice T2*-weighted functional images were acquired with a gradient-echo echo-planar imaging sequence using axial slice orientation and covering the whole brain (32 slices; voxel size: $3 \times 3 \times 3 \mathrm{~mm}^{3}$; matrix size $64 \times 64 \times 32$; repetition time $=2000 \mathrm{~ms}$; echo time $=$ $30 \mathrm{~ms}$; flip angle $=78^{\circ}$; field of view $=192 \times 192 \mathrm{~mm}^{2}$ ). The three initial volumes were discarded to avoid T1 saturation effects.

\section{Processing}

fMRI data preprocessing was performed using Statistical Parametric Mapping 8 (SPM8; http://www.fil.ion.ucl.ac.uk/ spm). Preprocessing steps included slice-time correction, realignment and adjustment for movement-related effects, coregistration of functional onto structural data, segmentation of structural data, spatial normalization into standard stereotactic Montreal Neurological Institute (MNI) space, and spatial smoothing with a Gaussian kernel of $8 \mathrm{~mm}$ full width at half-maximum. Further motion correction was applied using ArtRepair toolbox for $\mathrm{SPM}^{1}$ which corrects for small, large and rapid motions, noise spikes, and spontaneous deep breaths. Finally, linear regression of mean global BOLD signal, mean ventricular BOLD signal and mean white matter BOLD signals from each voxel was performed. Even if it is still a debated question, it could be argued that global signal regression (Macey et al. 2004) could induce spurious correlations in our analysis [e.g., (Murphy et al. 2009)]. However, it has been shown in Honey et al. 2009) that global signal regression is an important step to better reveal the correlation between structural and functional connectivities. Since this SC-FC link is the main focus of our paper, we regressed out the global signal.

\footnotetext{
$\overline{1}$ http://cibsr.stanford.edu/tools/human-brain-project/artrepair-soft ware.html.
} 


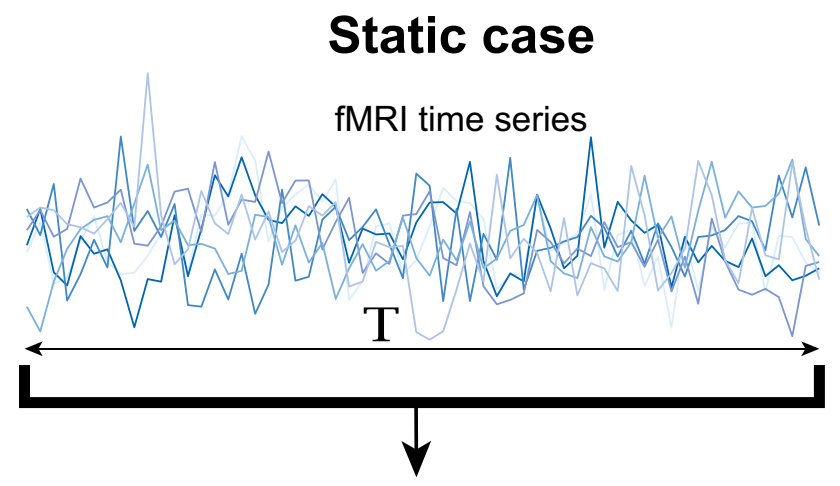

One Functional Connectivity matrix FC:

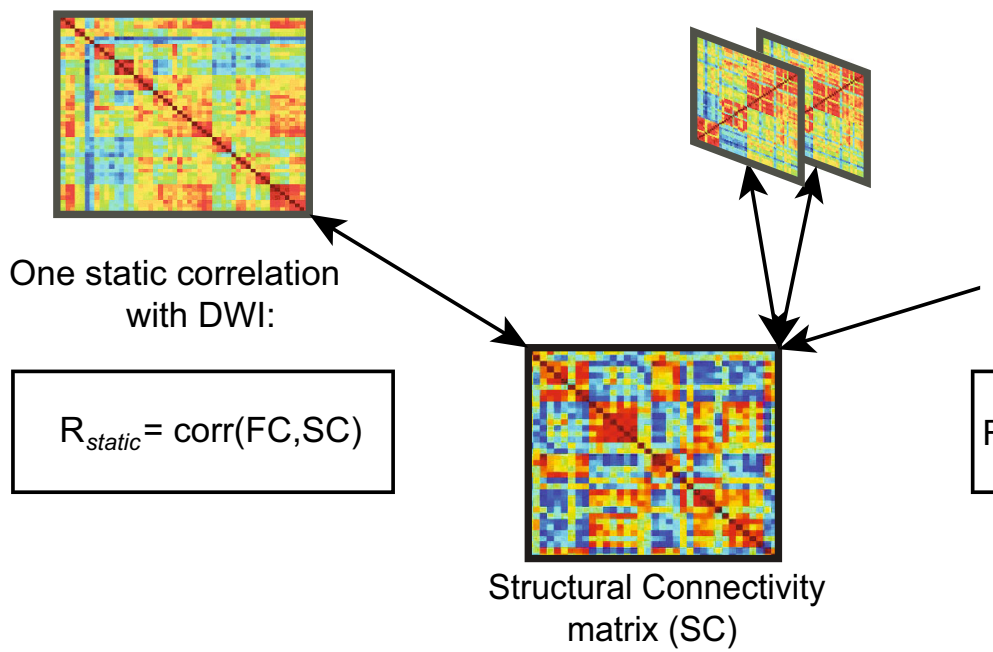

\section{Dynamic case}

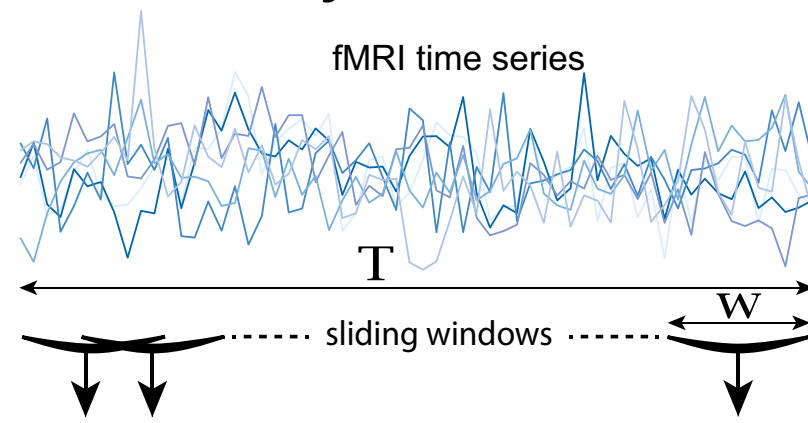

$\mathrm{T}-\mathrm{w}+1$ Functional Connectivity matrices $\mathrm{FC}(\mathrm{t})$ :

(dFC)

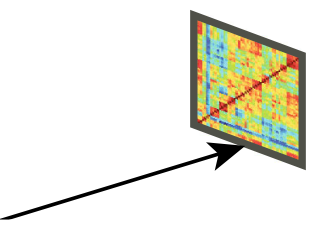

Dynamic correlation

with DWI:
Fig. 1 Comparison between the static and the dynamic analysis of the correlation between structural and functional connectivities (SC and FC, respectively). Left in the static case, FC is computed using the whole fMRI time course. The static correlation $R_{\text {static }}$ between SC and FC is then computed as in e.g., Honey et al. (2009). Right in the

The timecourse for each region-of-interest was extracted by taking the average signal over all voxels in each ROI defined following the same parcellation procedure as for anatomical data.

\section{Sliding window for FC analysis}

To explore the dynamics in the correlations between structural and functional connectivities, we repeated the computation of the FC matrices from truncated portions of the fMRI time series, as previously presented (Chang and Glover 2010; Hutchison et al. 2012; Allen et al. 2012; Leonardi et al. 2013). However, it has been shown recently that spurious correlations can arise from this classical approach when short windows are used. To limit this confound, we high-pass filtered the fMRI time series with a cutoff frequency of $1 / w$, where $w$ is the width of the truncated portions (Leonardi and Van De Ville 2015; Zalesky and Breakspear 2015). Denoting by $T$ the number dynamic case, $\mathrm{dFC}$ is computed in windows of the fMRI time courses that are slid across the whole fMRI time course. The dynamic correlation used in the present work $R_{\mathrm{dyn}}$ between $\mathrm{SC}$ and $\mathrm{FC}(t)$ is then computed and normalized by $R_{\text {static }}$ and $R_{\mathrm{dyn}}$ is a vector of size $T-w+1$

of volumes in the fMRI time series and considering a window width $w$, we computed $T-w+1$ successive FC matrices from the truncated fMRI time series in each particular window, each one being shifted forward by one TR with respect to the previous one (Fig. 1 right). We used window widths ranging from 5 to 100 volumes, corresponding to $10-200 \mathrm{~s}$, to explore the dynamics between structural and functional connectivities.

\section{Dynamic correlation between structural and functional matrices}

We then computed the correlation between all the FC matrices and the SC matrix (Fig. 1 right). This included, as in Honey et al. 2009), log-rescaling of the non-zero values in the SC matrix such that the range in both connectivity matrices has the same order of magnitude (see Supplementary Material for further details). To facilitate intersubject comparisons, the evolution of this correlation was 
normalized by the static correlation between the SC and the FC matrices computed using the whole fMRI time series (Fig. 1 bottom right), resulting in what we call the dynamic correlation, denoted by $R(t)$. To characterize the fluctuations of the dynamic correlation, the power spectrum of $R(t)$ was computed using Welch's method (Welch 1967) and normalized such that $\int_{0}^{0.25} P(f) d F=1$ where $P(f)$ is the power spectral content corresponding to frequency $f$.

\section{Values of interest}

To characterize dynamics observed in the time-evolving dynamic correlation curves and their corresponding spectral power, we used two markers:

- $V$ is the range of variation in the dynamic correlation, computed as the difference between its maximal and minimal value, in percent. $V$ is used to highlight the phases of (de)synchronization between SC and FC (Fig. 2a),

- $F^{*}$ is the frequency of maximal relative spectral power (in $\mathrm{Hz}$ ), and corresponds to the main oscillatory mode of a time course, such as in Fig. $2 b$.

\section{Statistical significance of the observed dynamics}

A key challenge in fMRI time series analyses is disentangling the neuronal dynamics from noise (Handwerker et al. 2012). To this end, we performed the same computations as the one described in Fig. 1 using surrogate data obtained by phase randomization in the Fourier domain of the fMRI volumes (Theiler et al. 1992), similar to what is presented in Allen et al. 2012), for example (see Supplementary Material for details). Doing so leaves the static correlation unchanged because the overall covariance structure is preserved, whereas the evolution of the dynamic correlation $R(t)$ using windowing will be totally rearranged.
We observed larger fluctuations (higher $V$ ) of $R(t)$ in the original data compared to the surrogate data. Hence, we chose this marker to test for differences between the results obtained with ordered and phase-randomized fMRI time series. For each value of window width and each subject, we did 1000 permutations [see e.g., Chap 3.5 in (Edgington and Onghena 1969)] and computed the $z$ score corresponding to the following null hypothesis:

$$
\mathcal{H}_{0}=\left\{V_{\text {ord }} \ngtr V_{\text {rand }}\right\}
$$

where $V_{\text {rmord }}$ (respectively, $V_{\text {rmrand }}$ ) is the range of variation of $R(t)$ in the original ordered (respectively, surrogate) data.

The group-level significance curve presented in Fig. 3b was computed from the $z$ scores of all the subjects. This technique is known as the Stouffer's method (Stouffer et al. 1949), and is detailed in the Supplementary Material.

\section{Graph theory metrics}

To further characterize FC during the phases of (de)synchronization with SC, we used three common graph metrics of FC considered as a weighted undirected graph (Bullmore and Sporns 2009). In this context, each ROI is considered as a node of the graph and the absolute value of the correlation between each two regions $i$ and $j, a b s\left(F C_{i, j}\right)$ is the weight of the edge connecting these two regions. Since FC is symmetric, it follows that the corresponding graph is undirected. We used the three following metrics on the whole FC matrices:

- Density is the number of total connections divided by the number of possible connections (Sporns 2002),

- Efficiency measures how 'close' every two nodes are in the graph. It is inversely related to the characteristic path length (Onnela et al. 2005; Rubinov and Sporns 2010),
A

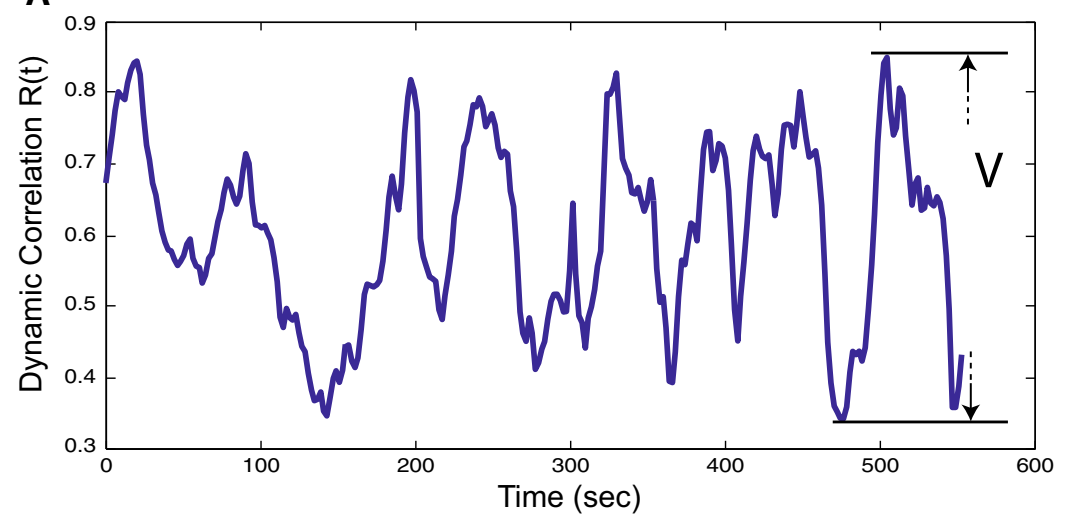

B

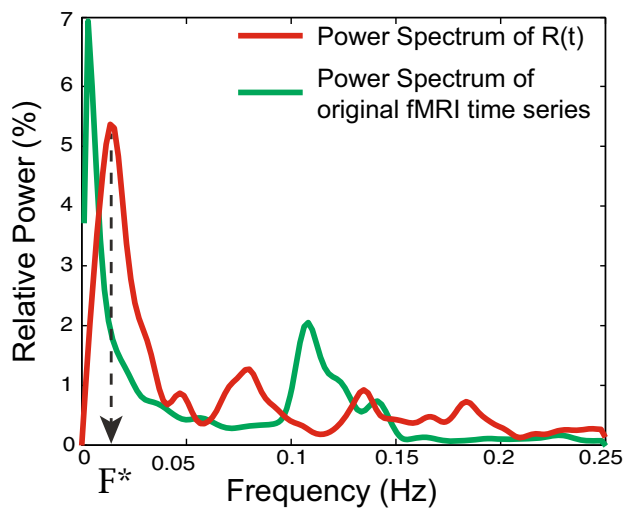

Fig. 2 a Temporal evolution of the dynamic correlation between SC and FC. b Corresponding power spectra. Results are shown for a representative subject and a window width of $w=13 \mathrm{TR}$. The static correlation $R_{\text {static }}=41.2 \mathrm{~V}=53.2 \%$ and $F^{*}=0.013$. The fMRI time series average spectrum for the same subject is also plotted for comparison 
A
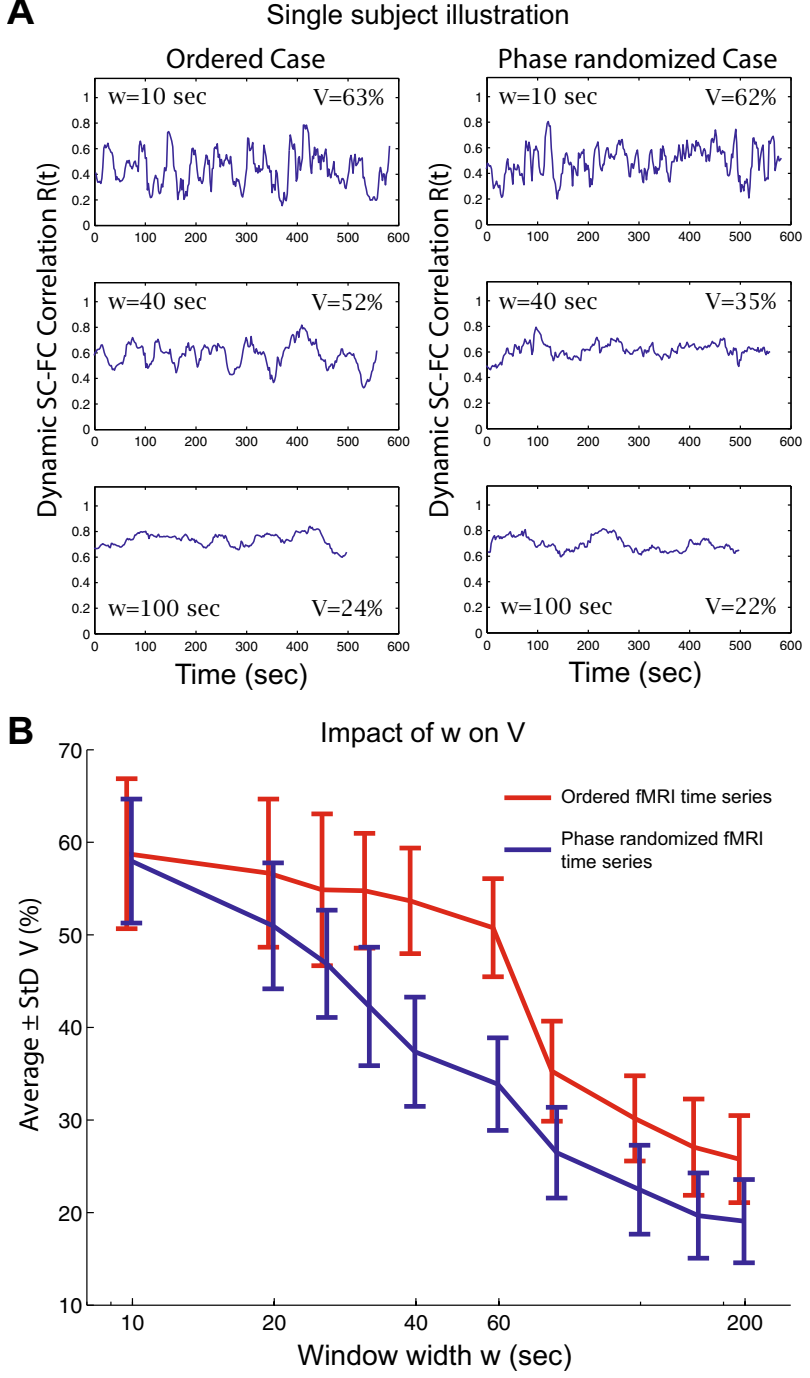

C

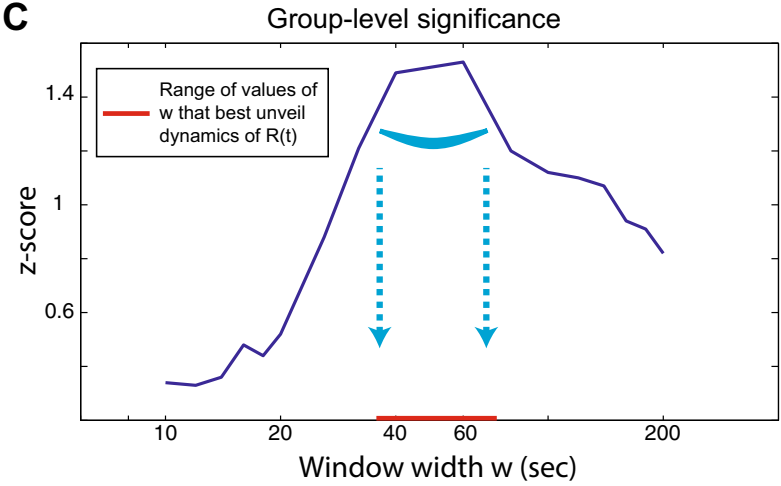

Fig. 3 a $R(t)$ for different window widths for a representative subject in the original dataset and one sample of the surrogate dataset. b Impact of the window width $w$ on $V$ at the group level, in ordered and phase-randomized time series. c Estimation of the statistical significance region at the group level based on $V$

- Modularity quantifies to which degree a network can be subdivided into distinct groups (Newman and Girvan 2004).
The Brain Connectivity Toolbox (Rubinov and Sporns 2010) was used to evaluate the value of these three markers during phases of high and low correlation between $S C$ and $F C(t)$. For each subject, averaged top and bottom $5 \%$ of $\mathrm{FC}(t)$ matrices were selected, sorted by $R(t)$ value. Since density was designed for binary graphs, we binarized the FC matrices (only for this marker) by considering only the significant correlations as defined by Leonardi and Van De Ville (2015), which corresponds in our case to a threshold of 0.44. It should be noted that the choice of these thresholds does not influence the trend observed in results presented in Fig. 5 as shown in Fig. S4.

For illustration purposes, ROIs belonging to auditory (AUD), somatomotor (SM), visual (VIS), cognitive control (CC), default mode (DM) were gathered in networks following the same grouping as in Allen et al. 2012) to highlight the modular and diagonal structures of the connectivity matrices.

\section{ROI structural and functional contributions}

All computations above have been performed at the wholebrain level. To explore the contribution of each of the 1015 regions to the brain level SC-FC dynamics, for each ROI we computed the dynamic correlation $R(t)$ (window width $w=20 \mathrm{TR}=20 \mathrm{~s}$ ) between SC and FC by considering only the connections implying this ROI. We then computed the range of variation $V$ of $R(t)$ as defined previously and computed the average variation over all subjects for each ROI. We finally also computed the average degree over the subjects of the corresponding regions of the structural connectivity matrix.

\section{Results}

\section{Statistical significance of dynamical correlation}

The dynamical correlation for a representative subject is shown in Fig. 2 for a window width $=20 \mathrm{TR}=40 \mathrm{~s}$. In this example, the dynamic correlation varies from 34 to $87 \%$ $(V=53 \%)$ of the static correlation, and the main oscillatory mode is $F^{*}=0.013 \mathrm{~Hz}$.

The choice of window width $w$ affects the way dynamical correlation is captured (see Fig. S1 in Supplementary Material). Significance of observed fluctuations as a function of $w$ is represented in Fig. 3 and was tested by comparison against phase-randomized fMRI time series as explained in the "Material and methods".

Figure $3 \mathrm{a}, \mathrm{b}$ illustrates the fact that the difference between ordered and phase-randomized fMRI time series as captured by $\mathrm{V}$ is more pronounced for intermediate values of $w$. At the group level, a peak of statistical 
significance can be observed around $w=20 \mathrm{TR}=40 \mathrm{~s}$ (Fig. 3c) hence this is the window width that we will use in the following analyses. It should be noted that the value of $V$ increases with the total length of the fMRI time series but stabilizes once this length is above 200-300 s. Hence, with a total acquisition time of $600 \mathrm{sec}$, the value of $\mathrm{V}$ is stable and the group-level curve presented in Fig. $3 \mathrm{c}$ is robust to changes in the recording time period (results not shown here).

\section{Phases of (de)synchronization between functional and structural connectivities}

We show in Fig. 4 the average patterns of $\mathrm{FC}(t)$ computed during phases corresponding to $5 \%$ highest and lowest value of $R(t)$ as well as the constant structural connectivity matrix, for one particular subject.

Density, efficiency and modularity of the high and low FC patterns for all the subjects are represented in Fig. 5.

Density and efficiency appear to be significantly lower $\left(p<10^{-4}\right.$ and $p<10^{-3}$ using a paired $t$ test) when the correlation between $\mathrm{FC}(t)$ and $\mathrm{SC}$ is high, whereas modularity increases at the same time $\left(p<10^{-3}\right)$.

\section{ROI-level analysis}

The average range of variation $V$ of $R(t)$ in the 1015 regions of interest is represented in Fig. 6a and the impact of the average degree of structural connectivity on $\mathrm{V}$ is represented in Fig. $6 b$.

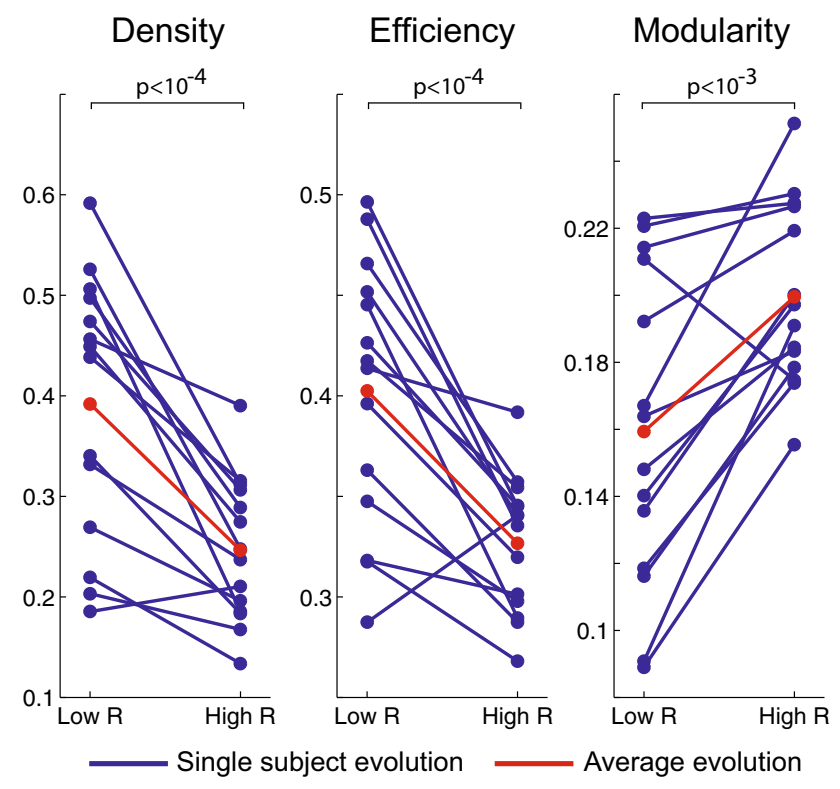

Fig. 5 Density, efficiency and modularity of FC averaged over the $5 \%$ lowest correlations with SC (low R left columns) and FC averaged over the $5 \%$ highest correlations with SC (high R right columns) for all the subjects. The group mean is represented in red

The regions with the highest values of $V$ are the ones that most contribute to the whole-brain level behavior presented in Fig. 3c. These areas encompass bilateral anterior cingulate, mesio-frontal, posterior cingulate, temporo-parietal and parahippocampal gyri that overlap with the default mode network, while the bilateral dorso-lateral prefrontal cortices are part of the executive control network (See "Discussion" for details). Note that also peri-rolandic

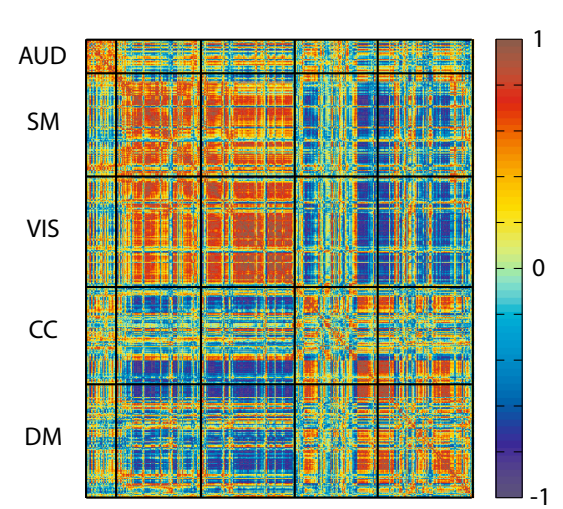

Average FC during phases of lowest FC-SC correlation

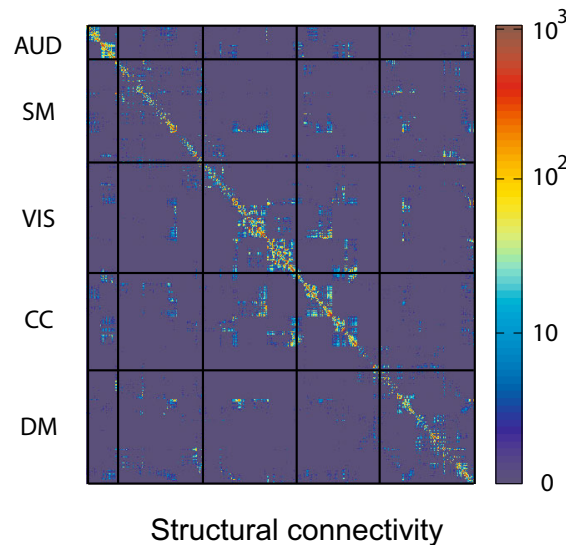

Structural connectivity

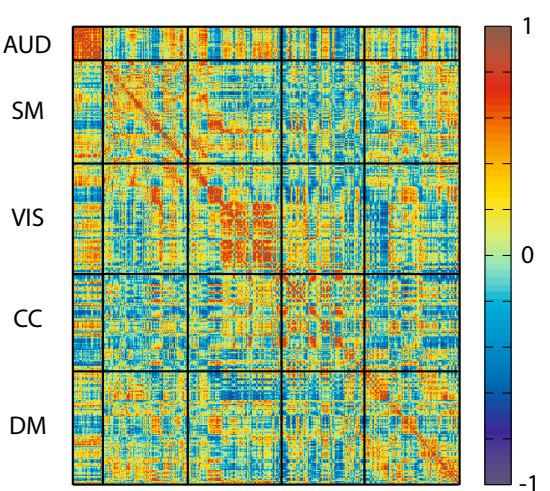

Average FC during phases of highest FC-SC correlation
Fig. 4 Left average FC matrix computed by averaging the FC matrices that have the $5 \%$ lowest correlations with SC for the same subject and $w=20 \mathrm{TR}=40 \mathrm{~s}$. Middle structural connectivity matrix. Right average FC matrix computed by averaging the FC matrices that have the $5 \%$ highest correlations with SC. Auditory
(AUD), somatomotor (SM), visual (VIS), cognitive control (CC), default mode (DM) networks are grouped to highlight the modular and block diagonal structure of connectivity matrices as in Allen et al. (2012) 


\section{A Left hemisphere \\ Right hemisphere}
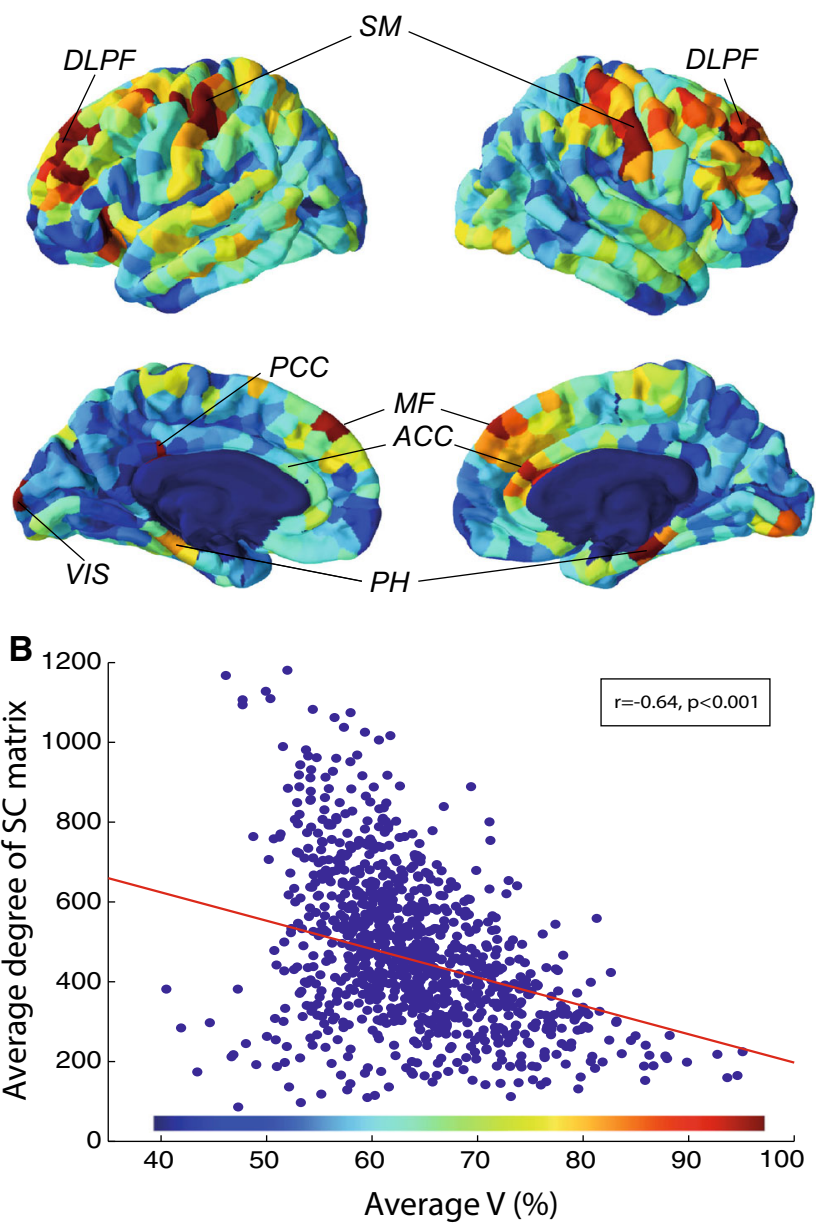

Fig. 6 a Average $V$ in each ROI for $w=40 \mathrm{~s}$ and corresponding distribution. $D L P F$ dorso-lateral prefrontal cortex, $S M$ sensory-motor cortex, $P C C$ posterior cingulate cortex, $M F$ mesio-frontal cortex, $A C C$ anterior cingulate cortex, $P H$ parahippocampal cortex, VIS visual cortex. b Link between average degree of SC matrix and average $V$

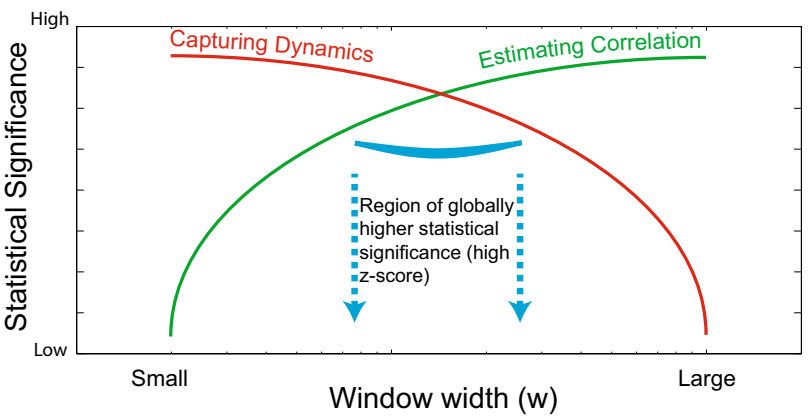

Fig. 7 Interpretation of results presented in Fig. 3c tradeoff between capturing dynamics and estimating correlation

sensory-motor and occipital visual sensory cortices showed a high range of variation of $R(t)$. Moreover, there is a significant negative correlation of 0.64 between the degree of the connectivity matrix and the value of $V$, averaged over the subjects.

\section{Discussion}

\section{Significance of fluctuations of $R(t)$ observed using the sliding window approach}

Sliding window techniques have been widely used in recent studies to analyze FC dynamics. Allen et al. (2012) used a width of $22 \mathrm{TR}$ (TR $=2 \mathrm{~s}$ ) to track oscillations in FC dynamics; Shirer et al. (2012) showed that considering a width above 15-30 TR (TR = 2 s) allows for robust estimation of the FC without considering dynamics. More recently, Leonardi et al. (2013) used widths ranging from 20 to 120 TR (TR $=1.1 \mathrm{~s}$ ) and observed different "eigenconnectivity patterns" depending on the window that was used and Hutchison et al. 2012) also found different results with window widths going from 10 to $120 \mathrm{TR}$ $(\mathrm{TR}=2 \mathrm{~s})$.

Our study reveals a peak of statistical significance in the observed fluctuations around $w=20-30$ TR $(\mathrm{TR}=2 \mathrm{~s})$ for which $w$ is sufficiently large to robustly estimate functional connectivity matrices and sufficiently small to capture corresponding dynamics. This results from a general tradeoff in time series analyses: longer windows improve the estimation of the correlation but mask the dynamics because they act as low-pass filters with cutoff frequency $f_{c}$ that decreases when $w$ increases (Smith 1997; Lindquist et al. 2014; Leonardi and Van De Ville 2015) and hence we can interpret the results of Fig. $3 b$ as the combination of these two effects is represented in Fig. 7.

The first effect illustrated by the green curve in Fig. 7 and corresponding to the estimation of functional connectivity was studied by Shirer et al. (2012). They show based on a classification experiment that using windows larger than 15-30 TR (TR = 2 s) allows for correct estimation of functional connectivity information, which is consistent with our findings. Our analysis provides the additional insight that considering higher values of $w$ does not capture the FC neuronal dynamics, which is illustrated by the red curve in Fig. 7. The peak in the ability to capture FC dynamics was observed using the range of variation $(V)$ of $R(t)$ and another statistical test based on the variance of the $R(t)$ curves (see Fig. S2 in Supplementary Material) also shows a peak for values of $w$ around 20 TR. Even if it is not surprising to find similar effects using variance instead of the range of variation, this confirms our conclusions about which window width should be used. Hence, we believe that the significance testing framework proposed in the present work is a simple way to find a window width $w$ that both allows for robust estimation of FC matrices and 
captures the corresponding dynamics. In addition, since this test is based on a comparison against phase-randomized time series, it allows to improve the interpretation of observed functional connectivity fluctuations and the delicate distinction from noise with similar properties (Hutchison et al. 2013) for which testing against generative null models might also be helpful for further characterization (Zalesky and Breakspear 2015). Let us finally note that the high-pass filtering preprocessing with cutoff frequency of $1 / w$ of the fMRI time courses performed following Leonardi and Van De Ville 2015) removes a large part of the original signal for small window widths. This may also explain why, for small values of $w$, the significance of the variations of $R(t)$ is poor (Fig. 3c). When $w$ increases in the range of 40-60 s, the effect of the highpass filtering becomes milder and the neuronal information expected to be encoded around $0.1 \mathrm{~Hz}$ is not filtered out leading to more significant fluctuations of $R(t)$. Finally, for higher values of $w$, the fluctuations of $R(t)$ are averaged and their statistical significance drops.

\section{Limitations}

The sliding window acts as a low-pass filter. In our case, considering $w=20 \mathrm{TR}=40 \mathrm{~s}$ results in a cutoff frequency $f_{c} \approx 0.02 \mathrm{~Hz}$ [Chap.15 (Smith 1997)]. Hence, a robust estimation of $\mathrm{FC}$, which requires a window width $w \approx 20$ TR, necessarily filters out the FC dynamics happening at higher frequencies than $\approx 0.02 \mathrm{~Hz}$. This limitation should be taken into account when interpreting results of dynamical FC analyses using sliding windows. In particular, it should be highlighted that having a main oscillatory mode at around $0.01 \mathrm{~Hz}$ (Fig. S1c in Supplementary Material) for the values of $w$ that are statistically significant does not mean that the dynamics are only occurring at these frequencies. As explained earlier, hypothetical dynamics happening at higher frequencies are filtered out when we use 20 TR (40 s) windowing. This is also a call for more advanced identification methods for estimating correlation, pushing the green curve of Fig. 7 to the smaller window widths, consequently freeing access to higher frequency dynamics. Finally, it is interesting to note that we were not able to distinguish ordered from phase-randomized time courses using $F^{*}$ (see Fig. S3 in the Supplementary Material), suggesting that $F^{*}$ is imposed by the sliding window method and by $w$ (Fig. S1c in the Supplementary Material) and is not a priori capturing neuronal dynamics. It is therefore not surprising to find similar values of $F^{*}$ in studies using a similar window width: Allen et al. (2012) found oscillations at $0.005-0.015 \mathrm{~Hz}$ using a 22 TR (44 s) windowing. However, as argued in Hutchison et al. (2013), this does not imply that the value of $F^{*}$ for ordered fMRI time series has a non-neuronal origin, which is why we decided to continue considering this marker, while remaining aware of this caveat.

\section{Phases of (de)synchronization between functional and structural connectivities}

The link between structural and functional connectivities was established a few years ago (Honey et al. 2009; van den Heuvel et al. 2009). Thereafter, a lot of interest has been devoted to deepening the understanding of how anatomical constraints shape functional connectivity (Honey et al. 2010; Breakspear et al. 2010; Cabral et al. 2011; Deco et al. 2012), and how this relationship can be affected by different pathologies (de Kwaasteniet et al. 2013; van Schouwenburg et al. 2013). In most of these studies, either the dynamics of FC are not taken into account, or it is modeled, but the information coming from the data and used to assess models is deduced with a static approach of FC [e.g., (Deco et al. 2013b)]. To our knowledge, our paper is the first purely data-driven attempt to study the dynamical relationship between SC and FC. More specifically, we show in Fig. 3 that there are statistically significant (i.e., resulting from the neuronal dynamics, not noise) phases of (de)synchronization between the functional correlation and the anatomical constraints. When using statistically significant values of $w$ such as $w=20 \mathrm{TR}$, the range of variation $V$ is in the order of $52 \%$ of the static correlation, compared to $35 \%$ in the randomized case, meaning that the correlation between FC and SC is significantly increased at some points and significantly decreased at some other points.

\section{Structure guides transitions between functional brain states}

Many studies have recently highlighted the presence of different and successive functional connectivity states, even at rest (Lv et al. 2013; Gao et al. 2010; Deco et al. 2013a; Yang et al. 2014; Sidlauskaite et al. 2014). The results shown in Figs. 4 and 5 suggest that the dynamic reorganization of functional connectivity patterns is at least partly shaped by anatomy. More particularly, it can be observed in Fig. 5 that phases of high correlation between FC and SC correspond to functional connectivity patterns that have low efficiency and high modularity. The interpretation is that during these phases the brain is poorly functionally connected, and organized in modules shaped by anatomy with few inter-module connections (Newman and Girvan 2004). On the other hand, during phases of low correlation between FC and SC, the number of intermodule functional connections increases, resulting in highly connected FC patterns. Very recently, Messé et al. 2014) demonstrated a decoupling between anatomically defined networks and other networks resulting from 
stationary and non-stationary FC dynamics, but not related to anatomy. Combined with our results, these observations lead us to propose that anatomy could periodically play the role of a relay that guides switches between different highly connected FC patterns not shaped by anatomy [low value of $R(t)$ ], alternating with phases of lower efficiency and higher modularity, defined by SC architecture [high value of $R(t)$ ]. Whereas we can consider the latter as similar modes of the $\mathrm{dFC}$ repertoire highly defined by structure, the FC patterns corresponding to low values of $R(t)$ may cover a much larger repertoire of FC modes, as previously observed (Gollo and Breakspear 2014; Shen et al. 2015), leading to a wider distribution of the graph metric values in Fig. 5. This interpretation echoes recent work (Zalesky et al. 2014) in which the most dynamic connections are shown to be inter-modular, and support the emergence of temporary phases of high functional efficiency. More generally, the spatial distribution of the level of fluctuation of dFC (Allen et al. 2012) and its link with cognitive tasks (Bassett et al. 2011) have also been explored. Interestingly, the anterior cingulate cortex, part of the DMN, was consistently identified as a region showing large dFC variability, coherently with the results presented in Fig. 6a. However, there does not seem to be a clear global correspondence between the regions identified in these studies and the ones identified here. One possible explanation for this might be the bias introduced in this work by the projection of $\mathrm{dFC}$ onto the underlying anatomical structure leading to a partial picture of $\mathrm{dFC}$ fluctuations. Furthermore, the results shown in Fig. 6b suggest that the level of the fluctuations is strongly influenced by the underlying anatomy, possibly explaining why the most stable functional connections are observed between symmetric inter-hemispheric ROIs, expected to be more densely connected, whereas the most variable connections are found between non-symmetric inter-hemispheric regions (Gonzalez-Castillo et al. 2014).

\section{$R(t)$ as a footprint of resting-state mentation?}

Our understanding of the physiological and cognitive meaning of FC as measured by resting-state fMRI is limited and controversial [e.g., (Lamme 2003; Vincent et al. 2007)]. Hence, the interpretation of the fluctuations of $R(t)$ remains speculative but we propose that they could be related to task-independent thoughts as classically reported during resting-state fMRI studies encompassing mind wandering, daydreaming, inner speech and mental imagery [for a review see Lieberman (2007)]. Indeed, growing neuroscientific evidence supports that these conscious mentation processes (Goldberg et al. 2006) are supported by the interplay between a default mode network (or internal awareness network) involved in self-related processes (Fox et al. 2005) with an external awareness network called the executive network (Vanhaudenhuyse et al. 2011). We now argue based on temporal and spatial arguments that $R(t)$ fluctuations could be related to these mentation processes. First, the temporal dynamics of our observed $R(t)$ possess a main oscillatory mode in the range $0.01 \pm 0.003 \mathrm{~Hz}$ (Fig. S1c in Supplementary Material). It is interesting to note the overlap with the so-called "periodic shifts" (Vanhaudenhuyse et al. 2011) from external to internal awareness of behavioral scores observed in healthy volunteers in resting-state fMRI conditions occurring with a frequency of $0.05 \mathrm{~Hz}$ (range $0.01-0.1 \mathrm{~Hz}$ ). However, this argument has to be considered cautiously because the fluctuations in spontaneous cognitive activity are known to show substantial variability at the individual subject level (Fox et al. 2007) and as indicated above we were not able to show that the frequency of the main oscillatory mode results from neuronal dynamics. Next, with regard to the spatial characteristics of the intensity of oscillations in $R(t)$, our results presented in Fig. 6 show that the identified bilateral anterior cingulate, mesio-frontal, posterior cingulate, temporo-parietal and parahippocampal gyri overlap with the default mode internal awareness network [e.g., (Kucyi and Davis 2014)], while the bilateral dorso-lateral prefrontal cortices are part of the executive control/external awareness control networks (Vanhaudenhuyse et al. 2011). Finally, let us note that these hypotheses could be linked to the previous point of the discussion. Indeed, Doucet et al. (2012) reported that mind wandering was correlated with fluctuations of functional modular organization, inner-oriented activities being associated to phases of low intermodule connectivity. This is an additional argument supporting the interpretation of $R(t)$ as reflecting task-independent processes encompassing mind wandering, but that could also be related to spontaneous thoughts (Buckner and Carroll 2007) or monitoring of the external world [for review see Hahn et al. (2007)].

\section{Future work}

The results of this paper call for several methodological refinements. Windowing is an approach that shows some limitations, and in our view future studies should consider more advanced techniques for identification of dynamics to further bridge the gap between the temporal dynamics of FC changes, their underlying static anatomical architecture, and their subjective cognitive counterparts. These alternatives should allow for clearer identification of dynamical changes in functional connectivity, and could help unveil processes occurring at higher frequencies, such as mind wandering. As fast scanning becomes feasible with new scanners and parallel imaging, one simple way to test this hypothesis would be to use smaller TRs, up to the sub- 
second range, to increase the low-pass cutoff frequency of the windowing process. It could also be worth completing the present multimodal analysis with other imaging modalities such as EEG. It has, for example, been suggested that EEG micro-states can be considered as building blocks of cognition, and that they shape the networks detectable with resting fMRI (Van de Ville et al. 2010). Hence, including the high-resolution temporal information provided by EEG measurements could lead to a better understanding of the interaction between anatomy and function and its interpretation in terms of cognitive processes.

\section{Conclusions}

The contribution of the present paper is twofold. From a methodological point of view, we highlight some characteristics of the sliding window technique to reveal functional connectivity dynamics. Our results suggest that the width of those windows should be chosen around the 20-30 TR (40-60 s) range to both provide a robust estimate of $\mathrm{FC}(t)$ and capture significant corresponding neuronal dynamics. For smaller or larger values, we could not distinguish functional connectivity dynamics from noise with similar properties. Next, we use a suitable window width to show that dynamical functional connectivity oscillates between states of high modularity, mostly shaped by structural connectivity architecture, and states of low modularity, not defined —or indirectly defined-by structural connectivity, during which more inter-network connections take place. Finally, considering that the fluctuations of $\mathrm{FC}(t)$ on the anatomical architecture are occurring at a characteristic frequency of $\approx 0.01 \mathrm{~Hz}$ and that regions mostly contributing to their dynamics belong to the default mode or the executive control networks, we propose that they could be related to task-independent processes such as mind wandering.

\section{References}

Allen EA, Damaraju E, Plis SM, Erhardt EB, Eichele T, Calhoun VD (2012) Tracking whole-brain connectivity dynamics in the resting state. Cereb Cortex 24:663-676

Amico E, Gomez F, Di Perri C, Vanhaudenhuyse A, Lesenfants D, Boveroux P, Bonhomme V, Brichant JF, Marinazzo D, Laureys S (2014) Posterior cingulate cortex-related co-activation patterns: a resting state FMRI study in propofol-induced loss of consciousness. PLoS One 9:e100012

Bassett DS, Wymbs NF, Porter MA, Mucha PJ, Carlson JM, Grafton ST (2011) Dynamic reconfiguration of human brain networks during learning. Proc Natl Acad Sci USA 108:7641-6

Bastian M, Sackur J (2013) Mind wandering at the fingertips: automatic parsing of subjective states based on response time variability. Front Psychol 4:573
Beckmann CF, DeLuca M, Devlin JT, Smith SM (2005) Investigations into resting-state connectivity using independent component analysis. Philos Trans R Soc Lond B Biol Sci 360:1001-13

Breakspear M, Jirsa V, Deco G (2010) Computational models of the brain: from structure to function. Neuroimage 52:727-30

Buckner RL, Carroll DC (2007) Self-projection and the brain. Trends Cogn Sci 11:49-57

Bullmore E, Sporns O (2009) Complex brain networks: graph theoretical analysis of structural and functional systems. Nat Rev Neurosci 10:186-98

Cabral J, Hugues E, Sporns O, Deco G (2011) Role of local network oscillations in resting-state functional connectivity. Neuroimage 57:130-9

Cammoun L, Gigandet X, Meskaldji D, Thiran JP, Sporns O, Do KQ, Maeder P, Meuli R, Hagmann P (2012) Mapping the human connectome at multiple scales with diffusion spectrum mri. J Neurosci Methods 203:386-97

Chang C, Glover GH (2010) Time-frequency dynamics of resting-state brain connectivity measured with fmri. Neuroimage 50:81-98

Christoff K, Gordon AM, Smallwood J, Smith R, Schooler JW (2009) Experience sampling during fMRI reveals default network and executive system contributions to mind wandering. Proc Natl Acad Sci USA 106:8719-24

Damoiseaux JS, Greicius MD (2009) Greater than the sum of its parts: a review of studies combining structural connectivity and resting-state functional connectivity. Brain Struct Funct 213:525-33

Damoiseaux JS, Rombouts SARB, Barkhof F, Scheltens P, Stam CJ, Smith SM, Beckmann CF (2006) Consistent resting-state networks across healthy subjects. Proc Natl Acad Sci USA 103:13848-53

Deco G, Hagmann P, Hudetz AG, Tononi G (2013a) Modeling resting-state functional networks when the cortex falls sleep: local and global changes. Cereb Cortex 24:3180-3194

Deco G, Ponce-Alvarez A, Mantini D, Romani GL, Hagmann P, Corbetta M (2013b) Resting-state functional connectivity emerges from structurally and dynamically shaped slow linear fluctuations. J Neurosci 33:11239-52

de Kwaasteniet B, Ruhe E, Caan M, Rive M, Olabarriaga S, Groefsema M, Heesink L, van Wingen G, Denys D (2013) Relation between structural and functional connectivity in major depressive disorder. Biol Psychiatry 74:40-7

Deco G, Senden M, Jirsa V (2012) How anatomy shapes dynamics: a semi-analytical study of the brain at rest by a simple spin model. Front Comput Neurosci 6:68

Desikan RS, Ségonne F, Fischl B, Quinn BT, Dickerson BC, Blacker D, Buckner RL, Dale AM, Maguire RP, Hyman BT, Albert MS, Killiany RJ (2006) An automated labeling system for subdividing the human cerebral cortex on MRI scans into gyral based regions of interest. Neuroimage 31:968-80

Doucet G, Naveau M, Petit L, Zago L, Crivello F, Jobard G, Delcroix N, Mellet E, Tzourio-Mazoyer N, Mazoyer B, Joliot M (2012) Patterns of hemodynamic low-frequency oscillations in the brain are modulated by the nature of free thought during rest. Neuroimage 59:3194-200

Edgington E, Onghena P (1969) Randomization tests. CRC Press

Engel J Jr, Thompson PM, Stern JM, Staba RJ, Bragin A, Mody I (2013) Connectomics and epilepsy. Curr Opin Neurol 26:186-94

Eryilmaz H, Van De Ville D, Schwartz S, Vuilleumier P (2011) Impact of transient emotions on functional connectivity during subsequent resting state: a wavelet correlation approach. Neuroimage 54:2481-91

Fox KCR, Nijeboer S, Solomonova E, Domhoff GW, Christoff K (2013) Dreaming as mind wandering: evidence from functional neuroimaging and first-person content reports. Front Hum Neurosci 7:412 
Fox MD, Snyder AZ, Vincent JL, Corbetta M, Van Essen DC, Raichle ME (2005) The human brain is intrinsically organized into dynamic, anticorrelated functional networks. Proc Natl Acad Sci USA 102:9673-8

Fox MD, Snyder AZ, Vincent JL, Raichle ME (2007) Intrinsic fluctuations within cortical systems account for intertrial variability in human behavior. Neuron 56:171-84

Friston KJ (2011) Functional and effective connectivity: a review. Brain Connect 1:13-36

Gao W, Zhu H, Giovanello K, Lin W (2010) Multivariate networklevel approach to detect interactions between large-scale functional systems. Med Image Comput Comput Assist Interv 13:298-305

Goldberg II, Harel M, Malach R (2006) When the brain loses its self: prefrontal inactivation during sensorimotor processing. Neuron 50:329-39

Gollo LL, Breakspear M (2014) The frustrated brain: from dynamics on motifs to communities and networks. Philos Trans R Soc Lond B Biol Sci 369:20130532

Gollo LL, Zalesky A, Hutchison RM, van den Heuvel M, Breakspear M (2015) Dwelling quietly in the rich club: brain network determinants of slow cortical fluctuations. Philos Trans R Soc Lond B Biol Sci 370:20140165

Gonzalez-Castillo J, Handwerker DA, Robinson ME, Hoy CW, Buchanan LC, Saad ZS, Bandettini PA (2014) The spatial structure of resting state connectivity stability on the scale of minutes. Front Neurosci 8:138

Gorgolewski K, Burns CD, Madison C, Clark D, Halchenko YO, Waskom ML, Ghosh SS (2011) Nipype: a flexible, lightweight and extensible neuroimaging data processing framework in python. Front Neuroinform 5:13

Greicius MD, Krasnow B, Reiss AL, Menon V (2003) Functional connectivity in the resting brain: a network analysis of the default mode hypothesis. Proc Natl Acad Sci USA 100:253-8

Griffa A, Baumann PS, Thiran JP, Hagmann P (2013) Structural connectomics in brain diseases. Neuroimage 80:515-26

Gusnard DA, Raichle ME, Raichle ME (2001) Searching for a baseline: functional imaging and the resting human brain. Nat Rev Neurosci 2:685-94

Hagmann P, Cammoun L, Gigandet X, Meuli R, Honey CJ, Wedeen VJ, Sporns O (2008) Mapping the structural core of human cerebral cortex. PLoS Biol 6:e159

Hahn B, Ross TJ, Stein EA (2007) Cingulate activation increases dynamically with response speed under stimulus unpredictability. Cereb Cortex 17:1664-71

Handwerker DA, Roopchansingh V, Gonzalez-Castillo J, Bandettini PA (2012) Periodic changes in fMRI connectivity. Neuroimage 63:1712-9

Hasenkamp W, Wilson-Mendenhall CD, Duncan E, Barsalou LW (2012) Mind wandering and attention during focused meditation: a fine-grained temporal analysis of fluctuating cognitive states. Neuroimage 59:750-60

Heine L, Soddu A, Gómez F, Vanhaudenhuyse A, Tshibanda L, Thonnard M, Charland-Verville V, Kirsch M, Laureys S, Demertzi A (2012) Resting state networks and consciousness: alterations of multiple resting state network connectivity in physiological, pharmacological, and pathological consciousness states. Front Psychol 3:295

Honey CJ, Sporns O, Cammoun L, Gigandet X, Thiran JP, Meuli R, Hagmann P (2009) Predicting human resting-state functional connectivity from structural connectivity. Proc Natl Acad Sci USA 106:2035-40

Honey CJ, Thivierge JP, Sporns O (2010) Can structure predict function in the human brain? NeuroImage 52:766-76

Hutchison RM, Womelsdorf T, Allen EA, Bandettini PA, Calhoun VD, Corbetta M, Della Penna S, Duyn JH, Glover GH,
Gonzalez-Castillo J, Handwerker DA, Keilholz S, Kiviniemi V, Leopold DA, de Pasquale F, Sporns O, Walter M, Chang C (2013) Dynamic functional connectivity: promise, issues, and interpretations. Neuroimage 80:360-78

Hutchison RM, Womelsdorf T, Gati JS, Everling S, Menon RS (2012) Resting-state networks show dynamic functional connectivity in awake humans and anesthetized macaques. Hum Brain Mapp 34:2154-2177

Jahanshad N, Rajagopalan P, Hua X, Hibar DP, Nir TM, Toga AW, Jack CR Jr, Saykin AJ, Green RC, Weiner MW, Medland SE, Montgomery GW, Hansell NK, McMahon KL, de Zubicaray GI, Martin NG, Wright MJ, Thompson PM (2013) Genome-wide scan of healthy human connectome discovers spon1 gene variant influencing dementia severity. Proc Natl Acad Sci USA 110:4768-73

Jones DT, Vemuri P, Murphy MC, Gunter JL, Senjem ML, Machulda MM, Przybelski SA, Gregg BE, Kantarci K, Knopman DS, Boeve BF, Petersen RC, Jack CR Jr (2012) Non-stationarity in the "resting brain's" modular architecture. PLoS One 7:e39731

Kaiser M (2013) The potential of the human connectome as a biomarker of brain disease. Front Hum Neurosci 7:484

Kiviniemi V, Vire T, Remes J, Elseoud AA, Starck T, Tervonen O, Nikkinen J (2011) A sliding time-window ICA reveals spatial variability of the default mode network in time. Brain Connect $1: 339-47$

Koch MA, Norris DG, Hund-Georgiadis M (2002) An investigation of functional and anatomical connectivity using magnetic resonance imaging. Neuroimage 16:241-50

Kötter R, Sommer FT (2000) Global relationship between anatomical connectivity and activity propagation in the cerebral cortex. Philos Trans R Soc Lond B Biol Sci 355:127-34

Kucyi A, Davis KD (2014) Dynamic functional connectivity of the default mode network tracks daydreaming. Neuroimage 100:471-480

Lamme VA (2003) Why visual attention and awareness are different. Trends Cogn Sci 7:12-18

Leonardi N, Richiardi J, Gschwind M, Simioni S, Annoni JM, Schluep M, Vuilleumier P, Van De Ville D (2013) Principal components of functional connectivity: a new approach to study dynamic brain connectivity during rest. Neuroimage 83:937-950

Leonardi N, Van De Ville D (2015) On spurious and real fluctuations of dynamic functional connectivity during rest. Neuroimage 104:430-6

Lieberman MD (2007) Social cognitive neuroscience: a review of core processes. Annu Rev Psychol 58:259-89

Lindquist MA, Xu Y, Nebel MB, Caffo BS (2014) Evaluating dynamic bivariate correlations in resting-state fmri: a comparison study and a new approach. Neuroimage 101:531-46

Liu X, Duyn JH (2013) Time-varying functional network information extracted from brief instances of spontaneous brain activity. Proc Natl Acad Sci USA 110:4392-7

Lv P, Guo L, Hu X, Li X, Jin C, Han J, Li L, Liu T (2013) Modeling dynamic functional information flows on large-scale brain networks. Med Image Comput Comput Assist Interv 16:698-705

Macey PM, Macey KE, Kumar R, Harper RM (2004) A method for removal of global effects from fmri time series. Neurolmage 22:360-6

McIntosh AR, Gonzalez-Lima F (1994) Structural equation modeling and its application to network analysis in functional brain imaging. Hum Brain Mapp 2:2-22

Messé A, Rudrauf D, Benali H, Marrelec G (2014) Relating structure and function in the human brain: relative contributions of anatomy, stationary dynamics, and non-stationarities. PLoS Comput Biol 10:e1003530

Moussa MN, Steen MR, Laurienti PJ, Hayasaka S (2012) Consistency of network modules in resting-state fmri connectome data. PLoS One 7:e44428 
Murphy K, Birn RM, Handwerker DA, Jones TB, Bandettini PA (2009) The impact of global signal regression on resting state correlations: are anti-correlated networks introduced? NeuroImage 44:893-905

Newman MEJ, Girvan M (2004) Finding and evaluating community structure in networks. Phys Rev E 69: 026113

Onnela JP, Saramäki J, Kertesz J, Kaski K (2005) Intensity and coherence of motifs in weighted complex networks. Phys Rev E 71:065103

Park HJ, Friston K (2013) Structural and functional brain networks: from connections to cognition. Science 342:1238411

Passingham RE, Stephan KE, Kötter R (2002) The anatomical basis of functional localization in the cortex. Nat Rev Neurosci 3:606-16

Richiardi J, Eryilmaz H, Schwartz S, Vuilleumier P, Van De Ville D (2011) Decoding brain states from fMRI connectivity graphs. Neuroimage 56:616-26

Rubinov M, Sporns O (2010) Complex network measures of brain connectivity: uses and interpretations. Neuroimage 52:1059-69

Sakoğlu U, Pearlson GD, Kiehl KA, Wang YM, Michael AM, Calhoun VD (2010) A method for evaluating dynamic functional network connectivity and task-modulation: application to schizophrenia. MAGMA 23:351-66

Shen K, Hutchison RM, Bezgin G, Everling S, McIntosh AR (2015) Network structure shapes spontaneous functional connectivity dynamics. J Neurosci 35:5579-88

Shirer WR, Ryali S, Rykhlevskaia E, Menon V, Greicius MD (2012) Decoding subject-driven cognitive states with whole-brain connectivity patterns. Cereb Cortex 22:158-65

Sidlauskaite J, Wiersema JR, Roeyers H, Krebs RM, Vassena E, Fias W, Brass M, Achten E, Sonuga-Barke E (2014) Anticipatory processes in brain state switching-evidence from a novel cuedswitching task implicating default mode and salience networks. Neuroimage 98C:359-365

Smith SM, Jenkinson M, Woolrich MW, Beckmann CF, Behrens TEJ, Johansen-Berg H, Bannister PR, De Luca M, Drobnjak I, Flitney DE, Niazy RK, Saunders J, Vickers J, Zhang Y, De Stefano N, Brady JM, Matthews PM (2004) Advances in functional and structural MR image analysis and implementation as FSL. Neuroimage 23(Suppl 1):S208-19

Smith SW (1997) The scientist and engineer's guide to digital signal processing, 2nd edn. California Technical Publications

Sporns O (2002) Graph theory methods for the analysis of neural connectivity patterns. Neuroscience databases. A Practical Guide. In: Kötter R (ed), pp 171-186

Sporns O, Chialvo D, Kaiser M, Hilgetag C (2004) Organization, development and function of complex brain networks. Trends Cogn Sci 8:418-425

Sporns O, Tononi G, Edelman GM (2000) Theoretical neuroanatomy: relating anatomical and functional connectivity in graphs and cortical connection matrices. Cerebral Cortex 10:127-141

Sporns O, Tononi G, Kötter R (2005) The human connectome: a structural description of the human brain. PLoS Comput Biol $1: \mathrm{e} 42$

Stouffer SES, DeVinney L, Star S, Williams R (1949) The American soldier, vol. 1: adjustment during army life. Princeton University Press
Tagliazucchi E, Balenzuela P, Fraiman D, Chialvo DR (2012) Criticality in large-scale brain FMRI dynamics unveiled by a novel point process analysis. Front Physiol 3:15

Theiler J, Eubank S, Longtin A, Galdrikian B, Farmer J (1992) Testing for nonlinearity in time series: the method of surrogate data. Physica D 58:77-94

Tournier JD, Calamante F, Connelly A (2007) Robust determination of the fibre orientation distribution in diffusion MRI: nonnegativity constrained super-resolved spherical deconvolution. Neuroimage 35:1459-72

Tournier JD, Calamante F, Connelly A (2012) MRtrix: diffusion tractography in crossing fiber regions. Int $\mathrm{J}$ Imaging Syst Technol 22:53-66

Tournier JD, Calamante F, Gadian DG, Connelly A (2004) Direct estimation of the fiber orientation density function from diffusion-weighted MRI data using spherical deconvolution. Neuroimage 23:1176-85

Vanhaudenhuyse A, Demertzi A, Schabus M, Noirhomme Q, Bredart S, Boly M, Phillips C, Soddu A, Luxen A, Moonen G, Laureys S (2011) Two distinct neuronal networks mediate the awareness of environment and of self. J Cogn Neurosci 23:570-8

Van de Ville D, Britz J, Michel CM (2010) EEG microstate sequences in healthy humans at rest reveal scale-free dynamics. Proc Natl Acad Sci USA 107:18179-84

Van den Heuvel MP, Mandl RCW, Kahn RS, Hulshoff Pol HE (2009) Functionally linked resting-state networks reflect the underlying structural connectivity architecture of the human brain. Hum Brain Mapp 30:3127-41

van Schouwenburg MR, Zwiers MP, van der Schaaf ME, Geurts DEM, Schellekens AFA, Buitelaar JK, Verkes RJ, Cools R (2013) Anatomical connection strength predicts dopaminergic drug effects on fronto-striatal function. Psychopharmacology (Berl) 227:521-31

Vincent JL, Patel GH, Fox MD, Snyder AZ, Baker JT, Van Essen DC, Zempel JM, Snyder LH, Corbetta M, Raichle ME (2007) Intrinsic functional architecture in the anaesthetized monkey brain. Nature 447:83-6

Welch PD (1967) The use of fast fourier transform for the estimation of power spectra: a method based on time averaging over short, modified periodograms. IEEE Trans Audio Electroacoust 15:70-73

Yang Z, Craddock RC, Margulies DS, Yan CG, Milham MP (2014) Common intrinsic connectivity states among posteromedial cortex subdivisions: insights from analysis of temporal dynamics. Neuroimage 93(Pt 1):124-37

Zalesky A, Breakspear M (2015) Towards a statistical test for functional connectivity dynamics. Neuroimage 114:466-70

Zalesky A, Fornito A, Cocchi L, Gollo LL, Breakspear M (2014) Time-resolved resting-state brain networks. Proc Natl Acad Sci USA 111:10341-6

Ziegler E, Foret A, Mascetti L, Muto V, Le Bourdiec-Shaffii A, Stender J, Balteau E, Dideberg V, Bours V, Maquet P, Phillips C (2013) Altered white matter architecture in BDNF met carriers. PLoS One 8:e69290 